\title{
A Histerossalpingo-sonografia como Método de Avaliação da Permeabilidade Tubária em Pacientes Inférteis
}

\author{
Hysterosalpingo-contrast Sonography in the Study of \\ Tubal Patency in Infertile Women
}

Angélica Lemos Debs Diniz, Alexandre Sérgio de Araújo Bezerra, João Fernando Kazan Tannus, José de Ávila Fernandes, Sílvia Caglieri Miguel, Vanessa Merjane.

\begin{abstract}
RESUM0
Objetivo: avaliar a histerossalpingo-sonografia (HSSG) como método alternativo no estudo da permeabilidade tubária em um grupo de pacientes inférteis.

Métodos: trata-se de estudo clínico transversal, que analisou a HSSG em comparação com a histerossalpingografia, como método de investigação da permeabilidade tubária, em um grupo de 31 pacientes inférteis. As tubas foram classificadas como: pérvias, ocluidas e inacessíveis. Foram determinadas a sensibilidade, especificidade e valores preditivos positivo e negativo da HSSG.

Resultados: a HSSG apresentou sensibilidade de 93,6\%, especificidade de 75\%, valor preditivo positivo de 95,7\%, valor preditivo negativo de 66,6\% e acurácia de $90 \%$. As trompas foram inacessiveis em 8,9\%. O tempo médio de execução do exame foi de 12 minutos. Dentre as pacientes que queixaram de dor durante a execução do exame 23,1\% referiram dor leve, 19,2\% dor moderada e 11,5\% dor intensa, porém 46\% das pacientes não referiram dor durante o exame.

Conclusão: a HSSG mostrou ser método seguro, altamente tolerável, de rápida execução, com boa sensibilidade e especificidade no estudo da permeabilidade tubárea em pacientes inférteis.
\end{abstract}

PALAVRAS-CHAVE: Permeabilidade tubária. Histerossalpingo-sonografia. Histerossalpingografia. Infertilidade.

\section{Introdução}

A determinação da permeabilidade tubária é parte fundamental na investigação de casais inférteis, tendo em vista que o fator tubário pode representar de 20 a 35\% das causas de infertilidade $^{1,2}$.

Tradicionalmente a investigação da permeabilidade tubária tem sido realizada utilizando-se dois métodos: a histerossalpingografia (HSG) e a cromotubagem via laparoscopia. A HSG, embora seja um método antigo, continua sendo largamente utilizada para o estudo das tubas

Universidade Federal de Uberlândia

Correspondência:

Angélica Lemos Debs Diniz

Al. João César de Souza, 110- Morada da Colina

38411-154 - Uberlândia - MG

e-mail: angelyca@net.em.com.br uterinas. Os dois principais incovenientes da HSG são a irradiação e o contraste iodado, que implicam alguns riscos ${ }^{3}$. Já a cromotubagem via laparoscopia é um método que permite a visualização direta da passagem de contraste (azul de metileno) para a cavidade abdominal após sua injeção através do colo uterino. A cromotubagem tem como inconveniente a necessidade de a paciente se submeter a laparoscopia e anestesia, levando aos riscos inerentes a estes procedimentos. Além disto, a cromotubagem via laparoscopia exige profissionais altamente qualificados e material específico, além de ter custos elevados ${ }^{2}$. Assim, nos deparamos com a necessidade de desenvolver um novo método acessível para investigação da permeabilidade tubária com baixos riscos e boa eficácia. A ultra-sonografia não foi esquecida por Nannini et $a .^{4}$, que foram os primeiros a descrever a histerossalpingo-sonografia (HSSG), com a 
injeção de soro fisiológico intra-útero, e que conseguiram estudar a permeabilidade tubária, obtendo resultados animadores. Três anos após, Richman et al. ${ }^{5}$ avaliaram 35 pacientes inférteis, realizando a HSSG com solução salina e obtiveram excelentes resultados na análise da permeabilidade tubárea. A partir destes primeiros estudos foi possivel otimizar o exame ultrasonográfico, no que diz respeito à análise da permeabilidade tubária. Desde 1986 existem à disposição no mercado meios de contraste para exames ultra-sonográficos baseados em suspensão de microgrânulos de galactose, que quando misturados e agitados produzem micro-bolhas que se aderem aos grânulos de galactose, produzindo imagem hiperecogênica ${ }^{6}$. Desde então tem-se tentado utilizar este contraste no lugar das soluções salinas, associado ao exame ultra-sonográfico transvaginal, para o estudo da permeabilidade tubária. Este seria um método simples, barato e sem os riscos descritos anteriormente para os outros exames ${ }^{7}$.

O objetivo deste estudo foi estudar a HSSG como método alternativo no estudo da permeabilidade tubária em um grupo de pacientes inférteis.

\section{Pacientes e Métodos}

Trata-se de estudo clínico transversal, realizado no Setor de Ultra-sonografia do Hospital de Clínicas da Universidade Federal de Uberlândia, que analisou a HSSG em comparação com a HSG, como método de investigação da permeabilidade tubária, em um grupo de 31 pacientes inférteis. As pacientes foram incluídas consecutivamente e os critérios básicos de inclusão foram a infertilidade primária ou secundária e a ausência de sinais clinicos de infecção ginecológica. Os critérios de exclusão foram a gravidez e sinais de infecção ginecológica, avaliados pelo exame clínico e citológico vaginal. Não foram usados antibióticos ou analgésicos antes e após os procedimentos. Todas as pacientes foram esclarecidas sobre o procedimento mediante termo de consentimento.

A média aritmética da idade foi de 29 anos, com variação entre 22 e 41 anos. Quanto ao número de gestações, 69,5\% das pacientes eram nuligestas, portanto portadoras de infertilidade primária, $13 \%$ e $17,5 \%$ eram primíparas e secundiparas, respectivamente, sendo portadoras de infertilidade secundária. Foi considerada infertilidade primária a ausência de gestação após 2 anos de vida sexual ativa, sem uso de métodos contraceptivos, e infertilidade secundária, em pacientes que tiveram uma ou mais gestações pregressas.

A avaliação ultra-sonográfica foi realizada por um único examinador, com o uso de equipamento Siemens Sonoline, versa Pro, transdutor transvaginal eletrônico, convexo, na freqüência de $6 \mathrm{MHz}$.

A HSSG foi realizada com a paciente em posição de litotomia. Antes do exame foi feita a antisepsia vaginal, introdução de cateter intra-útero e insuflação do balão com $1,5 \mathrm{ml}$ de soro fisiológico. A primeira solução a ser injetada era soro fisiológico para se estudar a cavidade uterina, seguido da injeção lenta do contraste Ecovist ${ }^{\mathbb{B}}$-Schering através do cateter, até se obter a visualização da extensão tubária e saída do contraste para o interior da cavidade peritoneal. A dopplerfluxometria foi utilizada no auxílio do diagnóstico da permeabilidade tubária em alguns casos, cuja linha hiperecogênica da tuba uterina não foi prontamente identificada no exame bidimensional. A seguir as pacientes eram encaminhadas imediatamente para a realização da HSG, com a injeção do contraste ioxitalamato de meglumina e de sódio (Telebrix $38^{\circledR}$ Guerbet) pelo mesmo cateter da HSSG. As HSG foram realizadas e interpretadas por um segundo examinador. Os meios de contraste foram fornecidos pela Universidade Federal de Uberlândia.

Quanto às características das tubas pela análise da HSSG, foram classificadas em pérvias, ocluídas e inacessiveis.

Solicitou-se às pacientes que informassem quanto à sintomatologia durante a realização da HSSG, baseado em escore subjetivo: sem dor-desconforto facilmente aceitável e mínimo durante o procedimento; dor leve - desconforto aceitável, equivalente a cólicas menstruais leves; dor moderada - desconforto com dor passivel de ser tolerada por curto período de tempo, e dor intensadesconforto intolerável, que compromete a realização do exame.

O tempo de execução da histerossalpingosonografia foi cronometrado a partir do início da colocação do espéculo vaginal para anti-sepsia cervical e vaginal, até a retirada do transdutor vaginal no final do exame.

Os resultados dos dois métodos em questão foram então confrontados para fins comparativos. Os examinadores não tiveram acesso prévio aos diagnósticos de cada método.

O método padrão-ouro utilizado neste estudo foi a HSG, baseado nas altas sensibilidade e especificidade citadas por vários autores e pela dificuldade de se realizar a cromotubagem via laparoscopia em todos os casos.

Os dados deste estudo foram apresentados 
de forma descritiva, utilizando-se porcentagem e média aritmética, conforme necessário. Foram determinadas a sensibilidade, especificidade, valores preditivos positivo e negativo e acurácia da HSSG.

\section{Resultados}

O único sintoma descrito pelas pacientes que se submeteram à HSSG foi a dor, que ocorreu em $53,9 \%$ dos casos. Na presença da dor, as pacientes referiram que a mesma foi leve, moderada e intensa em $23 \%, 19,2 \%$ e $11,5 \%$, respectivamente. Nesta amostra, 46,1\% das pacientes não referiram dor durante a realização da HSSG.

A duração média da HSSG foi de 12 minutos, com variação entre 7 e 19 minutos.

A análise do método foi realizada por tuba uterina, sendo estudadas 61 tubas, pelo fato de uma paciente ter se submetido a salpingectomia unilateral. Não foi possivel identificar seis tubas uterinas durante a injeção de Ecovist ${ }^{\circledR}$, portanto $8,9 \%$ de tubas foram classificadas como inacessíveis ao exame de HSSG. Quanto às seis tubas inacessíveis à HSSG tratava-se de quatro tubas pérvias mas desviadas cranialmente, uma tuba obstruída distalmente e uma tuba pérvia com hidrossalpinge, pelo estudo da HSG.

$\mathrm{Na}$ análise pela HSSG 43 tubas foram classificadas como pérvias e nove tubas como ocluídas, ao passo que, na análise da HSG, 44 tubas foram classificadas como pérvias e oito tubas como ocluídas (Tabela 1).

Tabela 1 - Análise da permeabilidade tubária feita pela histerossalpingo-sonografia e histerossalpingografia em um grupo de 31 pacientes inférteis.

\begin{tabular}{lccr}
\hline & $\begin{array}{c}\text { HSG tuba } \\
\text { pérvia }\end{array}$ & $\begin{array}{c}\text { HSG tuba } \\
\text { não-pérvia }\end{array}$ & Total \\
\hline HSSG: tuba pérvia & 44 & 2 & 46 \\
HSSG: não-pérvia & 3 & 6 & 9 \\
Total & 47 & 8 & \\
\hline
\end{tabular}

HSG: histerossalpingografia

HSSG: histerossalpingo-sonografia

A sensibilidade da HSSG na detecção de permeabilidade tubária foi de 93,6\% (IC: 0,70-1,16), com especificidade de $75 \%$ (IC: $0,56-0,93$ ), valor preditivo positivo de 95,6\% (IC: 0,71-1,19), valor positivo negativo de 66,7\% (IC: $0,50-0,83$ ) e acurácia de 90\% (Figura 1). Estas análises da permeabilidade tubária foram calculadas por tuba uterina avaliada, sendo descartadas as seis tubas uterinas inacessiveis ao exame.

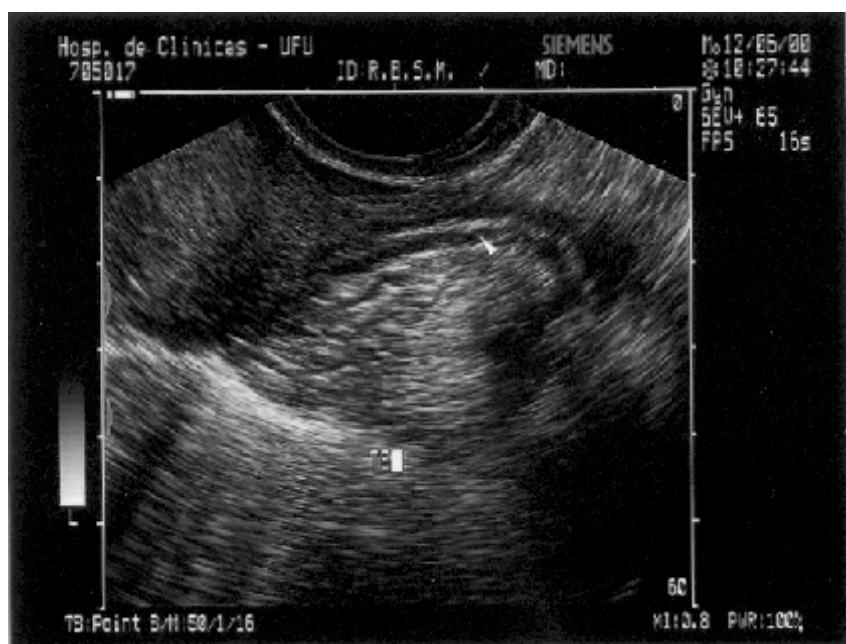

Figura 1 - Tuba classificada como pérvia na histerossalpingo-sonografia. Observa-se imagem hiperecogênica lateral ao útero, compatível com contraste no interior da tuba esquerda.

Identificamos três casos de hidrossalpinge na HSSG, em que o contraste preencheu parcialmente a tuba dilatada após a injeção de $20 \mathrm{~mL}$ de contraste, sendo observada uma tuba menos ecogênica devido à rarefação do contraste dentro da cavidade tubária alargada (Figura 2). Os diagnósticos de hidrossalpinge foram concordantes pelo uso da HSSG e da HSG.

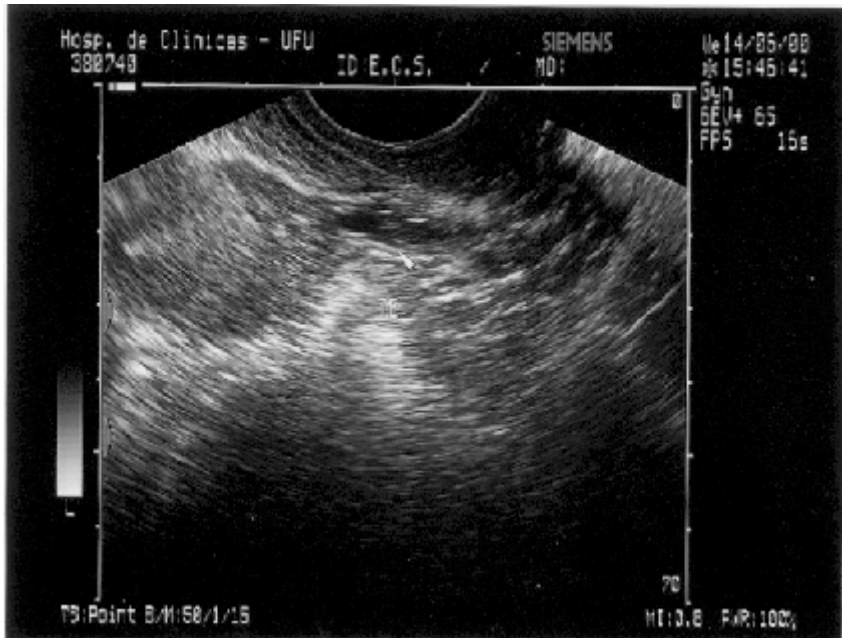

Figura 2 - Histerossalpingo-sonografia em um caso classificado como hidrossalpinge. Observa-se dilatação tubária com redução da ecogenicidade do contraste no interio da tuba esquerda.

\section{Discussão}

A investigação da permeabilidade tubária tem sido motivo de estudo constante no grupo de pacientes portadoras de infertilidade, pois a sua determinação é fundamental no que diz respeito às condutas a serem tomadas na programação te- 
rapêtica. Tendo em vista a necessidade de se desenvolver nova técnica menos invasiva, mais acessivel e eficaz na avaliação da permeabilidade tubária, foi proposto o estudo das trompas com o emprego de contrastes em conjunto com a ultrasonografia. A partir do emprego de novos contrastes na ultra-sonografia, a possibilidade de se obter sucesso no estudo da permeabilidade tubária tornou-se mais próxima de se concretizar. Estudos prévios já demonstraram que a HSSG seria um método seguro, bem tolerado e facilmente executado na rotina dos consultórios ultrasonográficos, além de ter boa acurácia se comparado à cromotubagem e à histero-salpingografia ${ }^{8-}$ 11. A HSSG tem como vantagens em relação aos outros métodos, o fato de proporcionar o estudo da cavidade uterina com a injeção de solução salina antes do contraste, além de fornecer mais informações sobre toda a anatomia pélvica (útero, ovários e fundo de saco posterior), o que não ocorre com a HSG.

Neste estudo a maioria das mulheres queixou-se de dor, embora a mesma fosse classificada como leve. Somente 11,5\% queixaram-se de dores intensas, o que está de acordo com os dados da literatura ${ }^{2,11}$. O tempo médio de execução da HSSG foi pequeno em relação aos outros métodos de análise da permeabilidade tubária e está de acordo com o tempo descrito pela maioria dos autores ${ }^{8}$.

A HSSG mostrou ser altamente sensivel no que diz respeito à análise da permeabilidade tubária, sendo compativel com grande parte dos trabalhos descritos na literatura, cujos métodos de comparação foram tanto a histerossalpingografia quanto a cromotubagem via laparoscopia $^{2,7,9,11,12}$. No que diz respeito à especificidade, encontramos valores inferiores aos descritos por alguns autores ${ }^{2,7,9,11,12}$, embora haja citações de baixa sensibilidade e especificidade da HSSG por um número reduzido de estudos ${ }^{6,13}$.

Uma das limitações da HSSG observadas neste estudo foi a dificuldade em se visibilizar a progressão do contraste em tubas uterinas desviadas cranialmente, devido à moderada penetração do som obtida pela sonda transvaginal, não alcançando, assim, toda a extensão da tuba desviada. Outra limitação foi a obstrução distal, cujo contraste progride parcialmente no interior da tuba, levando à falsa impressão de tuba pérvia. Neste último caso é fundamental que observemos a eliminação do contraste pela tuba distal, assim como o preenchimento do espaço peritoneal junto do fundo de saco posterior.

Sabemos que nenhum método diagnóstico pode assegurar acurácia absoluta no que diz respeito à permeabilidade tubária. O problema de todos os métodos é que estes podem definir a permeabilidade tubária com maior acurácia do que a sua oclusão. Se a tuba apresenta-se pérvia é muito provável que ela realmente seja pérvia, mas se a tuba apresenta-se obstruída, pode ser que tenha havido algum bias. As principais causas de bias no estudo da permeabilidade tubária são o extravazamento de contraste pelo colo, desvio cranial da tuba uterina, presença de fístula tubária, espasmo tubário e a recanalização espontânea.

Todos estes fatores deverão ser lembrados na análise dos resultados deste estudo.

Não nos parece que a HSSG irá substituir a HSG ou a cromotubagem, mas poderá reduzir a necessidade do uso dos métodos mais invasivos e da radiação no estudo da permeabilidade tubária. Não há dúvidas de que a HSSG ocupará seu espaço dentro do arsenal propedêutico do casal infértil, pela sua alta acurácia.

Concluímos que a HSSG mostrou ser um método seguro, altamente tolerável e de rápida execução, além de ser uma boa opção na avaliação inicial da permeabilidade tubária em pacientes portadoras de infertilidade.

\section{SUMMARY}

Purpose: to evaluate hysterosalpingo-contrast sonography as an alternative method in the study of tubal patency in a group of infertile women.

Methods: this is a transversal clinic study, which analyzed hysterosalpingo-contrast sonography and compared it with hysterosalpingography, as a method for the study of tubal patency, in a group of 31 infertile patients. Hysterosalpingocontrast sonography had a sensitivity of $93.6 \%$, specificity of $75 \%$, positive predictive value of $95.6 \%$, negative predictive value of $66.7 \%$ and accuracy of $90 \%$. The tubes were not accessible in $8.9 \%$. The mean time to perform the examination was 12 minutes. Fourty-six percent of the patients did not report pain during the examination and $23 \%$ reported light, $19.2 \%$ moderate, and only $11.5 \%$ reported severe pain.

Conclusion: hysterosalpingo-contrast sonography proved to be a safe, and tolerable method, of quick performance, with good sensitivity and specificity in the study of tubal patency in infertile women.

KEY WORDS: Hysterosalpingo-contrast sonography (HyCoSy). Infertility. Hysterosalpingography. Tubal patency. 


\section{Referências}

1. Kleinkauf-Houcken A, Hüneke B, Lindner C, Braendle W. Combining B-mode ultrasound with pulsed wave Doppler for the assessment of tubal patency. Human Reprod 1997; 12:2457-60.

2. Dietrich M, Suren A, Hinney B, Osmers R, Kuhn W. Evaluation of tubal patency hysterocontrast sonography (HyCoSy, Echovist) and its correlation with laparoscopic findings. J Clin Ultrasound 1996; 24:523-7.

3. Tanawattanacharoen S, Suwajanakorn S, Uerpairojkit B, Wisawasukmongchol W, Boonkasemsanti W, Virutamasen P. Transvaginal hysterosalpingo-contrast sonography (HyCoSy) compared with chromolaparoscopy: a preliminary report. J Med Assoc Thai 1998; 81:520-6.

4. Nannini R, Chelo E, Branconi F, Tantini C, Scarselli GF. Dynamic echohysteroscopy: a new diagnostic technique in the study of female infertility. Acta Eur Fertil 1981; 12: 165-71.

5. Richman TS, Viscomi GN, deCherney A, Polan ML, Alcebo LO. Fallopian tubal patency assessed by ultrasound following fluid injection. Radiology 1984; 152:507-10.

6. Ayida G, Harris P, Kennedy S, Seif M, Barlow D, Chamberlain P. Hysterosalpingo-contrast sonography (HyCoSy) using Echovist-200 in the outpatient investigation of infertility patients. Br J Radiol 1996; 69:910-3.
7. Cary WR. Note on determination of patency of the fallopian tubes by the use of collargol and X-ray shadow. Am J Obstet Gynecol 1914; 69:426.

8. Holz K, Becker R, Schürmann R. Ultrasound in the investigation of tubal patency. A meta-analysis of three comparative studies of Echovist-200 including 1007 women. Zentralbl Gynakol 1997; 119:366-73.

9. Reis MM, Soares SR, Cancado ML, Camargos AF. Hysterosalpingo-contrast sonography (HyCoSy) with SH U 454 (Echovist ${ }^{\circledR}$ ) for the assessment of tubal patency. Human Reprod 1998; 13:3049-52.

10.Ayida G, Chamberlain P, Barlow D, Koninckx P, Golding S, Kennedy S. Is routine diagnostic laparoscopy for infertility still justified? A pilot study assessing the use of hysterosalpingocontrast sonography and magnetic resonance imaging. Human Reprod 1997; 12:1436-9.

11.Kalogirou D, Antoniou G, Botsis D, Kassanos D, Vitoratos N, Zioris C. Is color Doppler necessary in the evaluation of tubal patency by hysterosalpingo-contrast sonography? Clin Exp Obstet Gynecol 1997; 24:101-3.

12.Stacey C, Bown C, Manhire A, Rose D. HyCoSy - as good as claimed? Br J Radiol 2000; 73:133-6.

13.Guerriero S, Ajossa S, Mais V, Paoletti AM, Melis GB. The screening of tubal abnormalities in the infertile couple. J Assist Reprod Genet 1996; 13:407-12.

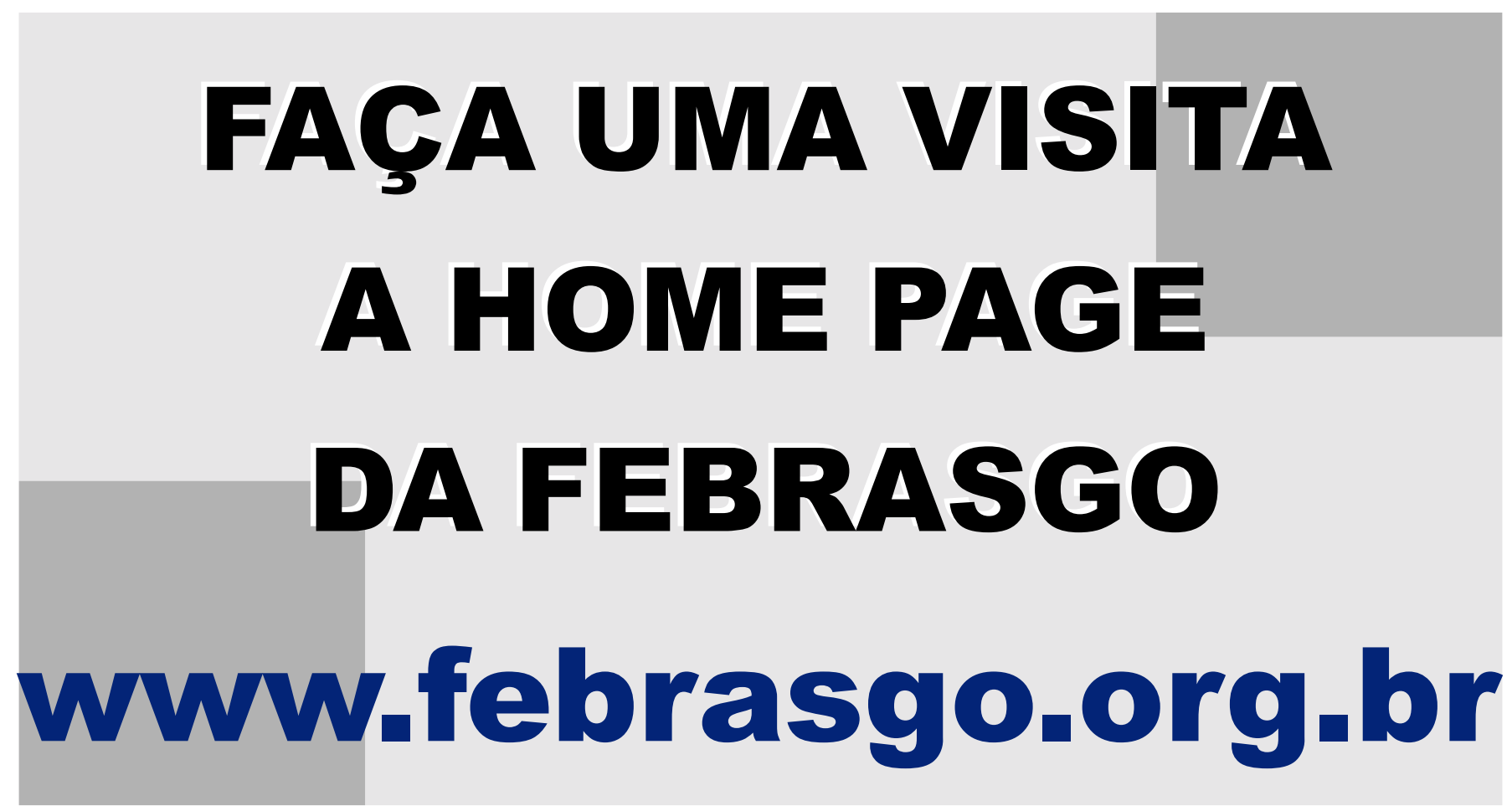

